\title{
Possible Impact of Select Trace Mineral Deficiency in HIV Seropositive Pregnant Women with/without Malaria Co-infection in NAUTH, Nnewi, Nigeria
}

\author{
Ikechukwu U. Chukwuagwu ${ }^{1}$, Nkiruka R. Ukibe ${ }^{1^{*}}$, Innocent S. Ogbu ${ }^{1}$, \\ Victoria O. Agu ${ }^{1}$, Joseph C. Awalu ${ }^{1}$, Friday A. Ehiaghe ${ }^{1}$ \\ and Obiageli B. Onyema-lloh ${ }^{2}$ \\ ${ }^{1}$ Department of Medical Laboratory Science, College of Health Sciences, Nnamdi Azikiwe University, \\ Nnewi Campus, P.M.B 5001, Nnewi, Anambra State, Nigeria. \\ ${ }^{2}$ Department of Chemical Pathology, College of Health Sciences, Nnamdi Azikiwe University, \\ Nnewi Campus, P.M.B 5001, Nnewi, Anambra State, Nigeria. \\ Authors' contributions \\ This work was carried out in collaboration among all authors. Author NRU conceptualized and \\ designed the study. Authors IUC, ISO, VOA and OBOI managed the literature searches and \\ performed the experiment. Authors IUC, VOA, JCA and FAE analyzed and interpreted the data. \\ Authors IUC and NRU wrote the original manuscript. All authors read and approved the final \\ manuscript. \\ Article Information \\ DOI: 10.9734/ARRB/2020/v35i530225 \\ Editor(s): \\ (1) Dr. Bechan Sharma, University of Allahabad, India. \\ (2) Paola Angelini, University of Perugia, Italy. \\ Reviewers: \\ (1) Muhammad Fawad Rasool, Bahauddin Zakariya University, Pakistan. \\ (2) Ekaete Francis Asuquo, University of Calabar, Nigeria. \\ (3) Karen Ingrid Tasca, IBB/UNESP, Brazil \\ Complete Peer review History: http://www.sdiarticle4.com/review-history/57305
}

Original Research Article

Received 30 March 2020

Accepted 06 June 2020

Published 20 June 2020

\section{ABSTRACT}

Aims: To evaluate the impact of HIV and malaria on serum Zinc (zn), Selenium (se) and Magnesium (mg) in HIV seropositive pregnant women in Nnewi, Nigeria.

Study Design: This is a case-control study.

Place and Duration of Study: Prevention of Mother to Child transmission (PMTCT) of HIV Clinic of Nnamdi Azikiwe University Teaching Hospital, Nnewi, (NAUTH), Nigeria, between December, 2017 and April, 2018. 
Methodology: We included 122 consented female participants; 32 HIV seropositive pregnant women with malaria parasitaemia, 30 HIV seropositive pregnant women without malaria parasitaemia, 30 Malaria infected pregnant women, 30 pregnant women without HIV or malaria parasitaemia, aged between 18 and 42 years. Screening for HIV antibodies and malaria parasite was double screened using National algorithm, rapid detection technique (RDT) and Giemsa staining microscopy technique respectively. Micronutrient levels were determined using atomic absorption spectrophotometry (AAS).

Results: When the mean $\mathrm{zn}$, sel and mg levels were compared between the test and control groups, significant decreases in serum zn (37.34 $\pm 6.7,36.70 \pm 4.39)$, sel $(29.93 \pm 6.02,28.00 \pm$ $5.04)$ and $\mathrm{mg}(2.41 \pm 0.46,2.33 \pm 0.14)$ were observed in HIV seropositive pregnant participants with/without malaria co-infection when compared with their corresponding controls $\mathrm{Zn}(45.1 \pm 7.83$, $50.36 \pm 8.73) ;$ Sel $(31.46 \pm 6.19,31.96 \pm 6.81) ; \mathrm{Mg}(2.62 \pm 0.30)$ and $(P<.05$ respectively $)$. Body mass index (BMI) was significantly decreased while diastolic blood pressure (DBP) and systolic blood pressure (SBP) were significantly increased in HIV seropositive pregnant women with malaria compared with their seronegative counterparts $(P<.05$ respectively).

Conclusion: The significant loss in BMI with derangement in serum zn, sel and mg levels in HIV seropositive pregnant women with/without malaria co-infection indicates high degree of malnutrition while the increased blood pressure suggests high blood pressure which might predispose the pregnant mothers to pre-eclampsia if not properly managed. Adequate micronutrient supplementation and effective anti malaria agent is strictly advocated in the routine management of HIV infected mothers during pregnancy to reduce the severity of the co-infection and adverse pregnancy complications especially in areas of malaria endemic transmissions.

Keywords: HIV/malaria; co-infection; trace minerals; pregnancy.

\section{INTRODUCTION}

Human immune deficiency virus and malaria infection remain a major public health issue in Nigeria health care system. Both infections pose great challenges in diagnosis and therapy specifically during pregnancy with detrimental outcomes that may affect both the mother and fetus [1]. It has been shown that HIV and malaria infections have a reciprocal effect on each other with a consequent increase in mortality rate [2]. HIV-infected pregnant women have been reported to be at greater risk of placental malaria infection [3]. Ayisi and colleagues has reported that malaria infection during pregnancy may increase the risk of mother-to-child transmission of HIV [4].

Trace elements deficiencies and HIV disease however, are thought to interact with each other [5]. The authors have shown that micronutrient deficiencies in HIV infected pregnant mothers could lead to disease progression and may contribute to an increased risk of placental malaria and adverse pregnancy outcomes [5]. The implications of these findings in malaria endemic region such as Nigeria form the bases for the present study.

\section{MATERIALS AND METHODS}

\subsection{Study Design}

This is a case-control study designed to assess the levels of trace elements (Zinc, Selenium, and Magnesium) in naive HIV seropositive subjects with malaria co-infection attending the ante-natal clinic at Nnamdi Azikiwe University Teaching Hospital in Nnewi (NAUTH). One hundred and twenty two (122) consented female participants aged between 18 and 42 years were randomly selected including: HIV seropositive pregnant women with malaria parasitaemia (32), HIV seropositive pregnant women without malaria parasitaemia (30), Malaria infected pregnant women without HIV infection (30), pregnant women without HIV or malaria parasitaemia (30) as control. All HIV seropositive pregnant women were yet to commence anti-retroviral therapy (naive). All subjects were screened for HIV seropositivity and malaria parasitaemia. Screening for HIV antibodies was done using Determine and Stat-Pak kit and confirmed with Unigold HIV kit. Pregnancy testing was done using human chorionic gonadotropin (HCG) one step pregnancy test strip. Peripheral malaria was double screened using Rapid Detection Technique (RDT) (2SD) and Giemsa stain thin and thick blood smears microscopy technique. 
A well-structured questionnaire was administered to each participant to obtain their reproductive history and other biodata. Levels of trace elements zinc, selenium and magnesium were determined using atomic absorption spectrophotometry.

\subsection{Study Site}

The study was conducted in Nnamdi Azikiwe University Teaching Hospital (NAUTH) in Nnewi, Anambra State, Nigeria. It is popularly known as NAUTH is located at the south eastern part of Nigeria. It is one of the oldest and most prestigious teaching hospitals in Nigeria and serves as a referral center for the nearby towns and states in the south eastern part of the country. Laboratory analysis of trace elements was done at Biotechnology Research Center, Nnamdi Azikiwe University, Awka.

\subsection{Subject Recruitment}

Purposive sampling technique was employed. The subjects were pregnant women visiting PMCT clinic at NAUTH between December, 2017 and April, 2018 who voluntarily agreed to participate and were subsequently enrolled in the study.

\subsection{Inclusion and Exclusion Criteria}

Pregnant participants between 18 and 42 years of age with HIV/or malaria infection were included in the study. Non-Pregnant participants seronegative to HIV with or without malaria infection were also included. Pregnant women less than 18 and above 42 years were excluded from the study. Participants who are active smokers, alcoholics, hypertensive and diabetic were excluded. Participant using zinc, selenium and magnesium supplement were also excluded.

\subsection{Sample Collection}

Five milliliters $(5 \mathrm{ml})$ of venous blood were collected from each of the participants and dispensed $2.5 \mathrm{ml}$ each into a well labeled plain container and an EDTA container. The plain bottle sample was allowed to clot and centrifugation was performed at $1500 \mathrm{rpm}$ for 5 minutes using bench centrifuge and serum separated for analysis of trace elements.

\subsection{Laboratory Analyses}

Determination of HIV-1/2 antibodies was done according to the National algorithm.
Malaria parasite screening was done using rapid detection test for Plasmodium falciparum malaria antigen as described by Murray and Gresser [6] and Giemsa stained thick and thin blood film for microscopic detection of $P$. falciparum parasites as described by WHO [7].

Determination of serum zinc, selenium and magnesium was done using atomic absorption spectrophotometry (AAS) as described by PerkinElmer [8].

\subsubsection{Anthropometric data collection}

A well-structured questionnaire was administered to all the participants to ascertain their medical history. The height and weight of the participants were measured. Height was measured to the nearest $0.1 \mathrm{~cm}$ using a tape rule, and weight measured to the nearest $0.1 \mathrm{~kg}$ using calibrated platform scales. The body mass index (BMI) was calculated by the formula of weight $(\mathrm{kg}) /$ height (m2).Obesity was assessed using body mass index (BMI) and waist circumference (WC). Three separate blood pressure (BP) readings were obtained per participants from the non-dominant arm using accoson sphygmomanometer with appropriate cuff size in sitting position at $2 \mathrm{~min}$ interval after an initial rest of 5-10 min with arm supported at heart level. The average of the past two readings was recorded for both systolic blood pressure and diastolic blood pressures (SBP and DBP) of each student. The cutoff value for hypertension was taken as SBP reading $\geq 130$ $\mathrm{mmHg}$ and/or DBP of $\geq 85 \mathrm{mmHg}$.

\subsection{Statistical Analysis}

Statistical package for social sciences (SPSS) version 22 was used for the statistical analysis. The data generated was analyzed using Analysis of variance (ANOVA) to compare more than two independent variables and student's t-test for two independent variables. Pearson correlation was used to correlate different parameters. Values were considered statistically significant if $p$ value $\leq .05$.

\section{RESULTS}

\subsection{Anthropometric Data}

When the BMI value was compared between the test and control groups, BMI value was significantly lower in HIV seropositive pregnant women with/without malaria co-infection (26.18 \pm $2.59,26.85 \pm 3.10$ ) compared with HIV 
seronegative pregnant women with/without malaria parasitaemia $(28.59 \pm 3.70,27.21 \pm$ 3.41) $(P=.001$ respectively).

When the mean DBP was compared between the test and control groups, DBP value was significantly higher in HIV seropositive pregnant women with malaria co-infection (85.53 \pm 9.90$)$, HIV seronegative pregnant women with malaria parasitaemia $(82.96 \pm 9.86)$ compared with HIV seropositive pregnant women without malaria parasitaemia (79.83 \pm 9.30) and HIV seronegative pregnant women without malaria parasitaemia $(77.60 \pm 5.75) \quad(P \leq .001$ respectively). The between group comparison showed that DBP value was significantly higher in HIV seronegative pregnant women with malaria parastaemia $(82.96 \pm 9.86)$ compared with HIV seronegative pregnant women without malaria parasitaemia (77.60 \pm 5.75$)$ $(P=.012)$.

When the mean SBP value was compared between the test and control groups, SBP value was significantly higher in HIV seropositive pregnant women with/without malaria parasitaemia $(133.18 \pm 8.18,125.4 \pm 6.71)$ compared with HIV seronegative pregnant women with/without malaria parasitaemia $(127.56 \pm 7.14,120.43 \pm 4.21)(P=.001$ respectively) (Table 1 ).

\subsection{Serum Zinc, Selenium and Magnesium Levels}

When the mean zinc level was compared between the test and control groups, the mean zinc level was significantly lower in HIV seropositive pregnant women with/without malaria co-infection $(37.34 \pm 6.7,36.70 \pm 4.39)$ compared with HIV seronegative pregnant women with/without malaria parasitaemia (45.1 \pm $7.83,50.36 \pm 8.73)(P=.001$ respectively $)$.

The mean level of selenium was compared between the test and control groups, selenium level was significantly lower in HIV seropositive pregnant women with/without malaria coinfection $(29.93 \pm 6.02,28.00 \pm 5.04)$ compared with HIV seronegative pregnant women with/without malaria parasitaemia $(31.46 \pm 6.19$, $31.96 \pm 6.81)(P=.044)$. The between group comparison showed that the mean level of selenium was significantly lower in HIV seropositive pregnant women without malaria parasitaemia $(28.00 \pm 5.04)$ compared with HIV seronegative pregnant women with/without malaria parasitaemia $(31.46 \pm 6.19,31.96 \pm 6.81)$ $(P=.033, .015$ respectively).

When the mean serum magnesium level was compared between the test and control participants, magnesium level was significantly lower in HIV seropositive pregnant women without malaria co-infection $(2.33 \pm 0.15)$ and HIV seronegative pregnant women with malaria parasitaemia $(2.27 \pm 0.14)$ compared with HIV seronegative pregnant women without malaria infection $(2.52 \pm 0.30)(P=.001)$. The between group comparison showed that magnesium level was significantly lower in HIV seronegative pregnant women with malaria co-infection (2.27 \pm 0.14) compared with HIV seropositive women with malaria parasitaemia $(2.33 \pm 0.15)(P=$ .002). Also, the mean magnesium level was significantly lower in HIV seronegative pregnant women with malaria parasitaemia $(2.27 \pm 0.15)$ compared with HIV seronegative women without malaria parasitaemia $(2.52 \pm 0.30)(P=.002)$ (Table 2).

\section{DISCUSSION}

Malaria infection seems to accelerate the degree of reduction in micronutrients status during pregnancy [9]. This condition can be worsened by combined effects of HIV infection as observed in the present study [10]. Zinc level was significantly decreased in HIV seropositive pregnant women with malaria co-infection when compared with control participants. This could be attributed to poor nutritional status and reduction in trace mineral levels among pregnant women. The finding was in agreement with previous report [11]. Hypozincemia has also been reported in patients with acute illnesses [12]. The reduction in zinc level observed in pregnant women has been implicated in many adverse pregnancy outcomes as a result of malaria infection [9]. Because of the role of zinc in immune modulation [13], it can be deduced that the significant reduction in zinc level in this study, is an indication of response to the challenges of oxidative stress caused by the triple effects of malaria parasites, possible inflammation as a result of HIV infection [14] as well as negative effect of pregnancy on the element.

The significant decrease in zinc level in HIV seronegative pregnant women without malaria parasitaemia compared with the non-pregnant HIV seronegative women without malaria parasitaemia confirmed the increased utilization of zinc in pregnancy for growth of the fetus. 
Table 1. Values of some anthropometric parameters in HIV seropositive pregnant women with/without malaria co-infection and control group

\begin{tabular}{|c|c|c|c|}
\hline & BMI(kg/m2) (mean+ SD) & $\mathrm{DBP}(\mathrm{mmHg})($ mean \pm SD) & SBP $(\mathrm{mmHg})($ mean \pm SD) \\
\hline HIV seropositive pregnant women with $m p(A) n=32$ & $26.18+2.59$ & $85.53 \pm 9.90$ & $133.18+8.18$ \\
\hline HIV sero-positive pregnant women without $m p(B) n=30$ & $26.85 \pm 3.10$ & $79.83 \pm 9.30$ & $125.4 \pm \overline{6} .71$ \\
\hline HIV seronegative pregnant women with $m p(C) n=30$ & $28.59 \pm 3.70$ & $82.96 \pm 9.86$ & $127.5 \overline{6}+7.14$ \\
\hline HIV seronegative Pregnant women without $\mathrm{mp}$ (Control) (D) $n=30$ & $27.21 \pm 3.41$ & $73.60 \pm 5.75$ & $120.43+4.21$ \\
\hline F- Value & $7.105^{-}$ & $9.68-$ & 17.02 \\
\hline P- Value type & 0.001 & 0.001 & 0.001 \\
\hline A VS B & 0.374 & 0.007 & 0.001 \\
\hline A VS C & 0.002 & 0.216 & 0.005 \\
\hline A VS D & 0.173 & 0.001 & 0.001 \\
\hline$B \vee S C$ & 0.024 & 0.137 & 0.277 \\
\hline$B \vee S D$ & 0.638 & 0.289 & 0.014 \\
\hline C VS D & 0.074 & 0.012 & 0.001 \\
\hline
\end{tabular}

$P$ was considered statistically significant if $\leq .05$

Table 2. Serum Levels of zinc, selenium and magnesium in HIV seropositive pregnant women with/without malaria co-infection and control groups

\begin{tabular}{|c|c|c|c|}
\hline & $\begin{array}{l}\text { Zinc (ug/dl) } \\
\text { (mean }+ \text { SD) }\end{array}$ & $\begin{array}{l}\text { Selenium(ng/ml) } \\
\text { (mean + SD) }\end{array}$ & $\begin{array}{l}\text { Magnesium (mg/dl) } \\
(\text { mean + SD) }\end{array}$ \\
\hline HIV seropositive pregnant women with $m p(A) n=32$ & $37.34+6.27$ & $29.93+6.02$ & $2.41+0 . \overline{16}$ \\
\hline HIV seropositive pregnant women without $m p(B) n=30$ & $36.70+4.39$ & $28.00+5.04$ & $2.33+0.15$ \\
\hline HIV seronegative pregnant women with $m p(C) n=30$ & $45.10+7.83$ & $31.46+6.19$ & $2.27+0.14$ \\
\hline HIV seronegative Pregnant women without mp. (Control) (D) $n=30$ & $50.36+8.73$ & $31.96 \pm 6.81$ & $2.52 \pm 0.30$ \\
\hline F- Value & 38.48 & 2.52 & 6.61 \\
\hline P- Value type & 0.001 & 0.044 & 0.001 \\
\hline A VS B & 0.747 & 0.224 & 0.283 \\
\hline A VS C & 0.001 & 0.336 & 0.065 \\
\hline A VS D & 0.001 & 0.203 & 0.186 \\
\hline$B \vee S C$ & 0.001 & 0.033 & 0.444 \\
\hline B VS D & 0.001 & 0.015 & 0.019 \\
\hline C VS D & 0.010 & 0.757 & 0.002 \\
\hline
\end{tabular}


The significant drop in trace element status of HIV seropositive participants with malaria coinfection may be a determinant in ascertaining factors that predisposes or determines severity of the disease in malaria endemic area. A high prevalence of malaria parasitemia among HIV infected individuals has been previously reported in Nigeria [14]. This means that the impact of the interaction of malaria and HIV will be most apparent in areas with HIV epidemic and malaria endemic areas such as Nigeria. Malaria parasite itself has been reported to contain a complete glutathione redox system especially glutathione reductase, which are very essential to their growth and development showing that inhibition of glutathione reductase in the malaria parasite with improved trace element supplementation represents an important approach to anti-malarial drug development [5].

In this study, selenium level was significantly lower among HIV seropositive pregnant women without malaria parasitaemia, compared with HIV seronegative pregnant women without malaria parasitaemia. This decrease is independent of malaria parasitaemia showing the place of selenium in HIV infection as well as in pregnancy. During pregnancy, a lot of stress is experienced physiologically and pathologically. The physiological stress is due to changes resulting from increased demands for nutrients, and changes in plasma volume [15]. These increased demands eventually lead to decrease in micronutrients including selenium levels and this likely increase fetal mortality risk [16]. Several authors have linked selenium deficiency in HIV infection to increased oxidative damage, cadiomyopathy and disease progression [17], indicating beneficial effect of antioxidant selenium in inhibition of HIV disease progression and vertical transmission $[5,15]$.

The significant increase in selenium level in parasitemic pregnant women may be due to increased mobilization of this element to fight inflammation caused by parasites' invasion [18]. Furthermore, immune status and selenium concentration are said to increase as parity increases [19]. Increase selenium concentration has also been reported to have some beneficial effects in the treatment of malaria in children [13]; hence we dare to say that increased selenium concentration in parasitemic pregnant women, which may be an inflammatory response, is beneficial to both the pregnant mother and the unborn child.
In this study, the significant decrease observed in mean level of magnesium in HIV seropositive pregnant women with/without malaria coinfection compared with HIV seronegative participants is an indication of magnesium deficiency disease conditions as well as in pregnancy. This increases the demand for adequate supplementation of micronutrient in disease state and pregnancy. Magnesium is essential for many relevant physiological functions, such as bone growth, heart rhythm, vascular tone, nerve function, muscle contraction and relaxation [20]. Variations in the concentration of $\mathrm{Mg}$ may be caused by $P$. falciparum malaria, malnutrition as well as malabsorbtion [21]. There has been a report of increasing demands of adequate magnesium status during pregnancy especially in those from disadvantaged backgrounds [22]. A previous study has also reported decreased adverse pregnant complications with magnesium supplementation [23].

From this study loss of body mass was very significant among HIV seropositive pregnant women with or without malaria co-infection. Consequently, among HIV infected persons, secondary infections or co-infection are also significant predictors of low gestational weight gain [22], possibly through secretion of proinflammatory cytokines. Loss of fat mass due to infection during pregnancy might represent a decrease in available substrate for fetal growth [23]. Furthermore, reduction in weight could be a risk factor for chorioamnionitis through impairment in specific immune responses [24], which increases the risk of pregnancy outcomes [25]. The differences in BMI as observed in this study might be that some of the HIV seropositive pregnant women may have been under stress compounded by their poor nutritional and socioeconomic status.

Significant increase in blood pressure observed in HIV seropositive pregnant women than control pregnant women may be an indication of possible exposure to hypertension and preeclampsia. This was in line with the previous finding [26]. The authors reported higher prevalence of hypertension in HIV infected than in the HIV uninfected population. The increasing blood pressure could also be related to HIV specific factors such as lipodystrophy, atherogenesis and cytokines activity [27]. Infection with the human immunodeficiency virus type 1 in pregnant women represents an independent risk factor for maternal mortality and adverse pregnancy outcome [28]. Previous 
report has shown that immune hyperactivity to paternal antigens has been hypothesized to play a role in the development of hypertension in pregnancy, and the immunosuppression caused by HIV could temper the immune response at the placental site and reduce placental vasoconstriction [29]. This potential protection may be a function of the intensity of immunosuppression and may depend on the severity of HIV disease and the use of antiretroviral therapy.

Notably, just as with the case of HIV seropositive pregnant women without malaria parasitaemia, in this study, there is a significant increase in the diastolic and systolic blood pressure in HIV seronegative pregnant women with malaria parasitaemia when compared with control. This is in line with an earlier work by Ndao and colleagues [30]. It has been established that in areas of stable endemic malarial transmission such as Nigeria, Plasmodium falciparum infection during pregnancy is usually asymptomatic [31]. This is characterized by sequestration of parasites in the placenta. Massive sequestration of parasites in the placenta leads to placental ischemia and other fatal complications [32]. However, in normal pregnancy, the earliest stages of development take place in a low oxygen environment- tissue hypoxa [33] and thereby, enhancing the release of reactive oxygen species. This has shown to enhance the release of ROS that are potentially damaging to the cardiovascular system [34]. Pathological stress is mainly due to disease conditions including malaria, HIV and hypertension. The effects of malaria in pregnancy have been well described. It has been documented that malarial infection during pregnancy is a major cause of adverse pregnancy outcomes and maternal complications [28, 31].

Oxidative stress has emerged in recent years as a suspected component in the pathogenesis of HIV disease [35]. Research has shown that even in the earliest stages of infection, a deleterious reductive-oxidative (redox) imbalance may occur [36]. Moreover, in response to malaria, HIV, pregnancy and other infections, phagocytic cells such as polymorphonuclear leucocytes and macrophages usually engage in respiratory burst in their attempt to destroy pathogens as a host cell-mediated immune response, with the depleting effect on CD4+T cells, yielding free radicals that react to yield ROS [37]. This shows that HIV and malaria co-infection causes additional oxidative stress in pregnant women.
Since the immune system is constantly stimulated and free radical production is higher than in healthy individuals, adequate intake of antioxidants such as selenium is therefore, critical in minimizing oxidative stress $[18,35]$. It has been noted that HIV seropositive pregnant women have a higher risk of developing severe malaria infection particularly in malaria endemic area with attendant immune dysregulation [31, 38]. This can contribute to the sequestering of infected erythrocytes, adhesion of platelets and mononuclear cells [35]. This mechanism of inflammation and sequestration gives rise to tissue stress and significant rise in diastolic and systolic blood pressure as confirmed in this study in HIV seropositive pregnant women with malaria co-infection compared to controls.

\section{CONCLUSION}

From the findings in this study, HIV and malaria co-infection impact serious significant derangements in micronutrient status (zinc, selenium and magnesium) of HIV seropositive pregnant women with malaria co-infection. There was a significant change in anthropometric parameters (DBP, SBP and BMI) in HIV seropositive pregnant women with malaria coinfection. This could predispose the affected individuals to adverse pregnancy outcomes, preenclampsia and hypertension if not adequately managed. It is therefore, strictly recommended that adequate micronutrient supplementations with antioxidant trace minerals be included in routine module for management of HIV seropositive pregnant women with or without malaria co-infection during their antenatal visit especially in regions of malaria endemicity.

\section{LIMITATION OF STUDY}

There was lack of analysis of the participants' food profile due to short duration of the study, Further longitudinal study is advocated for explore the participant food habits, since the concentration of these nutrients is directly associated with food habits; time of HIV infection/diagnosis, and viral load + CD4 T count, as all these points can also be crucial for nutrient reduction, etc.

\section{CONSENT}

All authors declare that 'written informed consent was obtained from the participants for publication of this research work. 


\section{ETHICAL APPROVAL}

"All authors hereby declare that all experiments have been examined and approved by the board of human research ethics committee of Nnamdi Azikiwe University Teaching Hospital, Nnewi, Anambra State (NAUTH/CS/66/Vol.10/194/2017/ 104) and have therefore been performed in accordance with the ethical standards laid down in the 1964 Declaration of Helsinki."

\section{ACKNOWLEDGEMENTS}

Authors wish to acknowledge all the pregnant women who voluntarily gave their written informed concept for success of this study.

\section{COMPETING INTERESTS}

Authors have declared that no competing interests exist.

\section{REFERENCES}

1. Kwenti TE, Malaria and HIV coinfection in sub-Saharan Africa: prevalence, impact, and treatment strategies. Res Rep Trop Med. 2018;9:123-36.

DOI: 10.2147/RRTM.S154501

2. Ezechi O, Petterson KO, Byamugisha J. HIVIAIDS, Tuberculosis, and Malaria in Pregnancy. J Pregnancy. 2012;140826.

DOI: $10.1155 / 2012 / 140826$.

3. Sanyaolu AO, Fagbenro-Beyioku AF, Oyibo WA, Badaru OS, Onyeabor OS, Nnaemeka Cl Malaria and HIV co-infection and their effect on haemoglobin levels from three health-care institutions in Lagos, southwest Nigeria. Afr Health Sci. 2013; 13:295-300.

DOI: 10.4l314/ahs.v13i2.14

4. Ayisi JG, van Eijk AM, Newman RD. Maternal malaria and perinatal HIV transmission, Western Kenya, Emerging Infect Dis. 2004;10:643-652.

5. Cosby AS, Kosuke K, Roland K, Wafaie WF. November the role of selenium in HIV infection. Nutri Rev. 2010;68:671-81.

6. Nandi S, Irlam J, Visser ME, Rollins NN. Micronutrient Supplementation in Pregnant Women with HIV Infection. Review. Cochrane Database Syst Rev. 2012;3: CD009755.

DOI: 10.1002/14651858.CD009755
7. Murray CK, Gasser RA, Magill AJ, Miller RS. Update on rapid diagnostic testing for malaria. Clin Microbiol Rev. 2008;21:97110.

8. WHO. Malaria parasite counting. Malaria standard operating procedure. 2016; MMSOP. 09.

9. Unger HW, Ashorn P, Cates JE.. Undernutrition and malaria in pregnancy a dangerous dyad?. BioMed Central Med. 2016;142.

10. Lindsay $\mathrm{KL}$, Buss $\mathrm{C}$, Wadhwa $\mathrm{PD}$, Entringer $S$. The interplay between maternal nutrition and stress during pregnancy: Issues and Considerations. Ann Nutr Metab. 2017;70:191-200.

11. Pathak P, Kapil U, Dwivedi SN, Singh R. Serum zinc levels amongst pregnant women in a rural block of Haryana State, India. Asian Pac. J Clin Nutr. 2008;17:276 -9 .

12. Besecker BY, Exline MC, Hollyfield J, Phillips G, Disilvestro RA. A comparison of zinc metabolism, inflammation and disease severity in critically ill infected and noninfected adults early after intensive care unit admission. Am J Clin Nutri. 2011;93: 1356-64.

13. Iribhogbe OI, Agbaje EO, Oreagba IA, Aina OO, Ota AD. Therapeutic potential of selected micronutrients in malaria: An in vivo study in Plasmodium berghei infected mice. Biol Med. 2012;4:193-210.

14. Vanessa Matinez Manfio VM, Tasca KI, Gatto M, Loureiro da Silva V, Golim MA, De Souza LR "HIV-Infected Pregnant Women: A Microbial Translocation and Inflammatory Status Discussion." AIDS Res Hum Retro. 2019;35:217-218

15. Lindsay $\mathrm{KL}$, Buss $\mathrm{C}$, Wadhwa PD, Entringer $S$. The Interplay between Maternal Nutrition and Stress during Pregnancy: Issues and Considerations. Ann Nutr Metab 70:191-200.

16. Roland K, Ferdinand M, Said A, Gernard IM, Julia LF, Donna S, et al. Randomized, double-blind, placebo-controlled trial of selenium supplements among HIV-infected pregnant women in Tanzania: effects on maternal and child outcomes, Am J Clin Nutr. 2008;87:1802-8.

17. Ogbodo SO, Okaka ANC, Nwagha U, Ejezie FE, Okafor CS. Oxidative stress in symptomatic malaria parasitemic pregnant women from Malaria Endemic Area 
of Nigeria. Am J Med Mcd Sci. 2014;4:1 68-74.

18. Bella SD, Grilli E, Cataldo MA, Petrosillo N. Selenium deficiency and HIV infection. Infect Dis Rep. 2010;2:e18.

DOI: 10.4081/idr.2010.e18

19. Ogbodo SO, Okaka NC, Nwagha UI. Anti-infective antioxidant minerals levels in uncomplicated pregnancy in some rural communities of South East Nigeria. J Med Neutrino Nutraceut. 2013;9:52-7.

20. Gernand AD, Schulze KJ, Stewart CP, West KP, Christian P. Micronutrient deficiencies in pregnancy worldwide: health effects and prevention. Nature Reviews Endocrinology. 2016;12(5): 274-289.

21. Jahnen-Dechent $W$, Ketteler M. Magnesium basics. Clin kidney J. 2012;5: 13-4.

22. Eduardo Villamor, Michele L. Dreyfuss, Ana Baylín, Gernard Msamanga, Wafaie W. Fawzi. Weight loss during pregnancy Is associated with adverse pregnancy outcomes among HIV-1 Infected Women, The Journal of Nutrition. 2004;134(60): 1424-1431

23. Mohammad $Y Y$, Yasir PK, Zulfiqar AB. Maternal mineral and vitamin supplementation in pregnancy. Expert Rev Obstet Gynecol. 2010;5:241-56.

24. Elaheh Z, Amal T. Effect of magnesium supplement on pregnancy outcomes: A randomized control trial. Adv Biomed Res. 2017;6:109.

DOI: $10.4103 / 2277-9175.213879$

25. Villamor E, Msamanga G, Spiegelman D, Peterson KE, Antelman G, Fawzi WW. Pattern and predictors of weight gain during pregnancy among HIV-1 infected women from Tanzania and HIV-related loss of body mass $\mathrm{J}$ Acquir Immune Def Syndr. 2003;32:560-9.

26. Galinsky R, Polglase GR, Hooper SB, Black MJ, Moss TJM. The Consequences of Chorioamnionitis: Preterm birth and effects on development. J Pregnancy. 2013;412831.

DOI: $10.1155 / 2013 / 412831$

27. Coloma AG, Alvarez AM, Roca-Cusachs $\mathrm{CA}$, et al. Prevalence of arterial hypertension and lipid profile in HIV patients. Medicina Clinica. 2008;131:6814.
28. Sattler FR, Qian D, Louie S. Elevated blood pressure in subjects with therapy on blood pressure in HIV-infected patients. A prospective study in a cohort of naive patients. AIDS. 2001;15:15.

DOI: 10.1097/00002030-200110190-

00013

29. Ukibe NR, Onyenekwe CC, Anojulu AA, Onwubuya E, Kalu AO, UKIBE SN. Impact of Plasmodium falciparum malaria infection on serum cortisol, adrenocorticotropic hormone, pregnancy associated plasma protein-A and alpha-fetoprotein in pregnant women at Nnewi. Int $\mathrm{J}$ Biol Chem Sci 2019;13:1222-1230.

DOI:https://dx.doi.org/10.4314/ijbcs.v13i3. 1

30. Malasundera RC, Larbalestier N, Smith $\mathrm{JH}$, de Ruiter A, McG Thom SA, Hughes AD Preeclampsia, antiretroviral therapy, and immune reconstitution. Lancet. 2002; 360:1152-4i.

31. Ndao A, Dumont N, Fievet S, Doucoure A, Gaye, Lehesran JY. Placental malarial infection as a risk factor for hypertensive disorders during pregnancy in Africa: A case-control study in an Urban Area of Senegal. West Afr Am J Epidemiol. 2009; 170:847-53.

32. Onyenekwe CC, Meludu SC, Dioka CE, Salimonu LS. Prevalence of asymptomatic malaria parasitaemia amongst pregnant women. Indian J Malarial. 2002;39:60-5.

33. Dorman E, Shulman C. Malaria in pregnancy. Mini-symposium: Infections in pregnancy. Curr trends Obstet Gynecol. 10:183-9.

34. Jauniaux E, Poston L, Burton GJ. Placental-related diseases of pregnancy: Involvement of oxidative stress and implications in human evolution. Hum Repro Update. 2006;12:747-55.

35. Baillie JK, Bates MG, Thompson AA, Waring WS, Partridge RW, Schnopp MF. Endogenous urate production augments plasma antioxidant capacity in healthy lowland subjects exposed to high altitude. Chest. 2007;131:1473-8.

36. Osuji FN, Onyenekwe CC, Ifeanyichukwu M, Ahaneku JE, Ezeani M, Ezeugwunne IP. Antioxidant activity in HIV and malaria co-infected subjects in Anambra State, southeastern Nigeria. Asian Pacific J Trop Med. 2012; 841-7. 
37. Clark IA, Budd AC, Alleva LM. Cowden WB. Human malarial disease: A consequence of inflammatory cytokine release. Malar J 2006;5:85.

DOI: $10.1186 / 1475-2875-5-85$
38. French $\mathrm{N}$, Nakiyingi J, Lugada E, Watera C, Whitworth JA., Gilks CF. Increasing rates of malarial fever with deteriorating immune status in HIV-1-infected Ugandan adults. AIDS. 2001;15:899-906.

(c) 2020 Chukwuagwu et al.; This is an Open Access article distributed under the terms of the Creative Commons Attribution License (http://creativecommons.org/licenses/by/4.0), which permits unrestricted use, distribution, and reproduction in any medium, provided the original work is properly cited.

Peer-review history:

The peer review history for this paper can be accessed here: http://www.sdiarticle4.com/review-history/57305 